\title{
On-board sample cleaver
}

\author{
Martin Månsson,, ${ }^{\text {a) }}$ Thomas Claesson, Ulf O. Karlsson, and Oscar Tjernberg \\ Materials Physics, Royal Institute of Technology KTH, S-164 40 Kista, Sweden \\ Stéphane Pailhés, Johan Chang, and Joël Mesot \\ Laboratory for Neutron Scattering, ETH Zürich and PSI Villigen, CH-5232 Villigen PSI, Switzerland
}

Ming Shi and Luc Patthey

Swiss Light Source, Paul Scherrer Institut, CH-5232 Villigen PSI, Switzerland

Naoki Momono, Migaku Oda, and Masayuki Ido

Department of Physics, Hokkaido University, Sapporo 060-0810, Japan

(Received 2 March 2007; accepted 18 June 2007; published online 18 July 2007)

\begin{abstract}
An on-board sample cleaver has been developed in order to cleave small and hard-to-cleave samples. To acquire good cleaves from rigid samples the alignment of the cleaving blade with respect to the internal crystallographic planes is crucial. To have the opportunity to mount the sample and align it to the blade ex situ has many advantages. The design presented has allowed us to cleave very tiny and rigid samples, e.g., the high-temperature superconductor $\mathrm{La}_{(2-x)} \mathrm{Sr}_{x} \mathrm{CuO}_{4}$. Further, in this design the sample and the cleaver will have the same temperature, allowing us to cleave and keep the sample at low temperature. This is a big advantage over prior cleaver systems. As a result, better surfaces and alignments can be realized, which considerably simplifies and improves the experiments. (C) 2007 American Institute of Physics. [DOI: 10.1063/1.2756754]
\end{abstract}

\section{INTRODUCTION AND MAIN IDEA}

In modern surface science the quality of the samples and, in particular, the surface has become increasingly important for the final results, e.g., in high resolution angle resolved photoemission spectroscopy (ARPES). In addition, the materials of interest in this field also tend to have particularly complex crystal structures, e.g., high-temperature superconductors (HTSCs), resulting in smaller and smaller single-crystalline samples. With today's modern synchrotrons it is possible to focus the beam to a very small spot i.e., to actually measure on small samples is not an obstacle. The real problem has, so far, been how to in situ attain a clean and flat surface. The oxides, including HTSCs, cannot be cleaned by ion-bombardment due to selective sputtering of the different elements. Instead one can either scrape the surface using a diamond file or, in the case of single-crystal samples, try to cleave the sample along a specific crystallographic plane. There are several available systems ${ }^{1,2}$ with a cleaver inside the ultrahigh vacuum (UHV) chamber, usually mounted on a wobble stick or a linear transfer. When it comes to oxides of simple structure, e.g., $\mathrm{NiO}$, where large single-crystal samples can be grown, there are no big problems to use these systems. Though when dealing with more complex oxides and HTSCs the samples are very small and "conventional" cleavers are almost impossible to use. Hence most HTSCs are instead cleaved using the so called "postcleave method" where a small metal post is glued to the sample surface and then "pushed" with a wobble stick inside vacuum in order to cleave (or rather break) the sample into

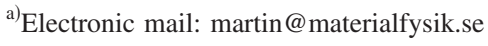

two halves. This method works rather satisfactorily on soft and layered structures, e.g., $\mathrm{Bi}_{2} \mathrm{Sr}_{2} \mathrm{CaCu}_{2} \mathrm{O}_{8+\delta}(\mathrm{Bi} 2212)$ but on more rigid samples such as $\mathrm{Nd}_{(2-x)} \mathrm{Ce}_{x} \mathrm{CuO}_{4}$ (NCCO) or $\mathrm{La}_{(2-x)} \mathrm{Sr}_{x} \mathrm{CuO}_{4}$ (LSCO) there are more severe problems. After a postcleave of these samples one often ends up with no cleave at all or in other cases a cleave but not along any specific crystallographic plane (i.e., a break and not a cleave).

As a remedy to shortcomings of available methods an on-board sample cleaver has been developed (see Fig. 1). The main idea is to have a miniature cleaver built into the sample carrier. It is hereby possible to align the desired cleavage plane of the sample to the blade of the cleaver by the use of, e.g., Laue x-ray diffraction (XRD) before introducing the sample into the UHV system. Another advantage is that very small samples can be cleaved since the position of the blade onto the sample can be aligned ex situ using a stereomicroscope. This not only gives the possibility to cleave both small and rigid samples, but also makes life a lot easier for the user. Finally, since the cleaver is mounted on the sample holder the sample and the blade have the same temperature, allowing us to cleave and keep the sample at low temperature.

\section{THE DESIGN}

The on-board cleaver was originally designed to be used at the SIS beamline ${ }^{3}$ of the Swiss Light Source (SLS). Hence, the available sample transfer system with a sample carrier having a diameter of only $16 \mathrm{~mm}$ and a usable height of merely $9 \mathrm{~mm}$ was used. As a result, the work to integrate a cleaver in this very limited space was quite a delicate matter [see Fig. 2(a)]. In addition, two different versions (Ver. 1 


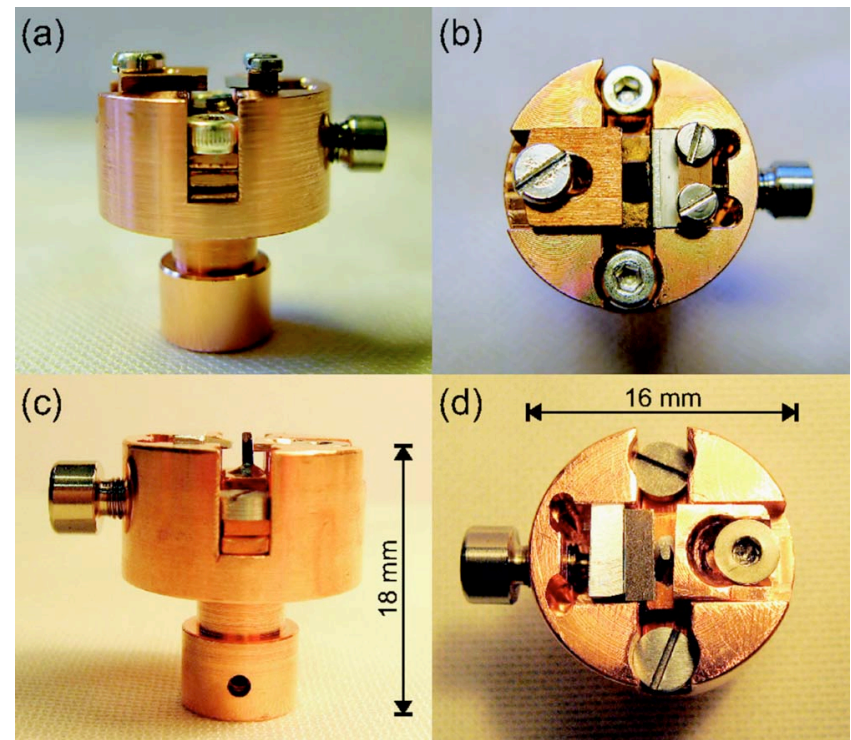

FIG. 1. (Color online) Photograph of the on-board sample cleaver: (a) side view Ver. 1, (b) top view Ver. 1, (c) side view ver. 2, and (d) top view Ver. 2

and Ver. 2) have been developed. Ver. 1 is a slightly more versatile and, regarding material choices, simpler (less expensive) version suitable for ARPES measurements under "normal" conditions. In Ver. 2 the design has been improved in order to provide a larger field of view, and it is also constructed from more "exotic" materials in order to avoid any stray magnetic fields when performing measurements at extremely low temperatures.

The design starting point was a blade moving along the sample carrier surface and towards its center where the sample is located. For mechanical stability a solution with a T-shaped slider in a milled track of the carrier was chosen. A replaceable blade is mounted to the slider using either two M1.5 bolts (Ver. 1), or through a simple tight-fit into a thin slit of the slider (Ver. 2). The latter has the advantage that no bolts protrude and hinder photons to reach the sample during experiments at grazing incidence. This design has a big advantage compared to preceding cleavers where a damaged blade only can be replaced by venting the UHV system. Since the blade would always work at a fixed height the sample height needed to be adjustable. The sample, which is usually mounted to the carrier using silver-epoxy glue, should also be in very good thermal contact with the carrier in order to be cooled to a temperature below $10 \mathrm{~K}$. Both problems were solved by simply stacking thin metal spacers on top of each other until the blade and sample are aligned at the desired height. This not only allows good thermal contact, but also acts as a very exact way of adjusting the height (compare "Johansson Gauge Blocks") making it possible to cleave even very thin (sub-1 mm) samples. Another advantage is that the same sample can be reused for a second cleave very easily by just adding an extra spacer and a new cleave of the same sample can be made. Everything is held together by two bolts that are mounted directly into threaded holes of the carrier. To facilitate good access to the sample at grazing incidence the top sample plate has a slightly elevated area dedicated to the sample. When cleaving glued samples there is always a risk that the sample will come loose due to the applied force. To minimize this risk an anvil is placed on the opposite side (with respect to the blade) of the sample.

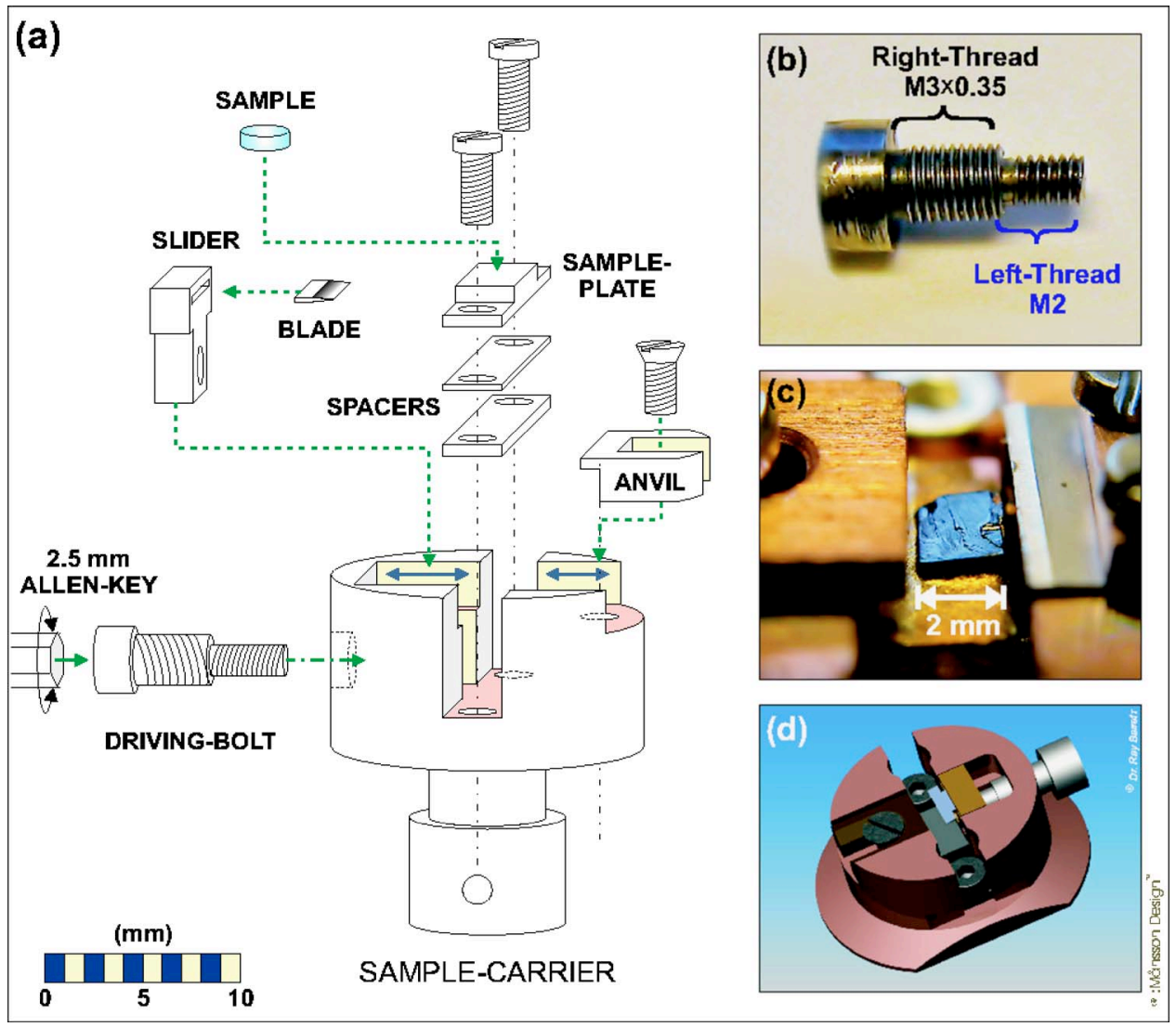

FIG. 2. (Color online) (a) Exploded view of the cleaver (Ver. 2) showing all individual parts. (b) Photograph of the double-threaded driving bolt. (c) Photograph of a test-cleaved LSCO sample. (d) Ver. 3 of the cleaver, designed for the ID08 beamline (Ref. 2) at the European Synchrotron Radiation Facility (ESRF). Courtesy of Dr. Ray Barrett (ESRF). 
The anvil can be slid into contact with the sample and then fixed by a bolt that is countersunk to facilitate good access to the sample.

When working in UHV the only connection between the outside and the sample inside is usually very simple tools connected via wobble sticks or linear transfers. Here the only available controlling aid was a $2.5 \mathrm{~mm}$ Allen key mounted on a wobble stick connected to the vacuum chamber of the ARPES end station. Hence the blade needed to be movable towards and away from the sample using a bolt with a $2.5 \mathrm{~mm}$ hexagonal head. A compact solution with a doublethreaded driving bolt [Fig. 2(b)] was developed. The part closest to the bolt head has a normal M3 thread that goes into a threaded hole of the carrier body, while the tip of the bolt is left (counterclockwise) threaded M2 that goes into a leftthreaded hole of the slider. By this simple solution, the slider can easily be moved in both directions. If the bolt is turned clockwise it will be screwed into the carrier body and at the same time screwed out of the slider. Both movements will push the slider towards the sample. On the other hand, if the bolt is turned counterclockwise the bolt will be screwed out of the carrier body and at the same time into the slider. Both movements will retract the slider from the sample, allowing the beam to access the complete sample surface. The slope of the M3 thread has been chosen to fit the slope of the M2. Hence the force applied will be distributed on equal amounts of "individual" threads in the carrier body and slider.

Our design allows a rather short $(2 \mathrm{~mm})$ movement of the blade, but one needs to recall that we are supposed to cleave the sample, not cut or saw! As a result, when cleaving, the blade will not be able to move across the whole sample and hereby scratch the surface. In other types of cleavers this is actually a problem that not everybody is aware of. Especially for less rigid samples where one might not immediately "feel" that the sample has cleaved and hence continues to move the blade forward.

Since the cleaver is incorporated into the sample carrier the materials used have to be carefully selected. Especially for Ver. 2, which is to be used in measurements of both energy and momentum distribution of photoelectrons at very high resolution. First, all materials need to be UHV compatible. Second, it is important to eliminate any stray magnetic fields and also taking into consideration that some measurements are to be made at very low temperatures where even, e.g., stainless steel can become magnetic. Consequently, the main sample carrier, spacers, sample plate, and anvil have all been manufactured in oxygen-free high conductivity (OFHC) copper that is completely nonmagnetic and also facilitate good thermal and electrical contact with the manipulator. The slider is made out of an aluminum alloy but Be bronze (a hardened copper alloy containing 2\% beryllium) has also been employed. Preferably, the slider should also be coated with a thin layer of gold in order to reduce the friction when sliding in the track of the copper sample carrier. This is crucial in vacuum where parts tend to adhere very well to each other due to the absence of moist and air, which ex situ work as "lubricants." When cleaving oxides it is essential that the blade is sharp and of very high hardness. Consequently, the blade can be made out of, e.g., tungsten, tungsten carbide, or even a standard razorblade (demagnetized). All bolts (including the driving bolt) are made out of either titanium or molybdenum.

\section{FUNCTIONALITY}

The on-board cleaver has been used in ARPES experiments where we have collected high quality data ${ }^{4-6}$ from the high-temperature superconductor $\mathrm{La}_{(2-x)} \mathrm{Sr}_{x} \mathrm{CuO}_{4}$. It should be noted that these samples are known to be very hard to cleave at all. Though assisted by the on-board cleaver we can now routinely perform successful cleaves of these and similar samples [see Fig. 2(c)]. In these low-temperature experiments $(T=15 \mathrm{~K})$ the advantage of having both the cleaver and sample at low temperature is evident. In prior cleaver systems the cleaver is usually at room temperature resulting in large temperature rise of the cooled sample (especially if the sample is small) when performing the cleave. In the case of, e.g., LSCO where the surface is known to rapidly decay, if heated, this is a major issue.

\section{DISCUSSIONS}

The on-board cleaver has proven to work exceptionally well in cleaving samples that have been a big problem to cleave prior to this design, e.g., LSCO. We can conclude that when the sample is carefully aligned ex situ using Laue $\mathrm{XRD}$, the probability of achieving a very nice cleave/surface is excellent. Further, this design allows us to cleave and keep the sample at low temperature since the sample and the cleaver will have the same temperature. Even though this design has been tailored for a specific system, the scheme of having a miniature cleaver incorporated into the sample carrier is a very versatile arrangement that can be adapted to a large variety of sample carriers. In fact, we have recently also developed a Ver. 3 for the ID08 beamline ${ }^{2}$ at the European Synchrotron Radiation Facility (ESRF). As shown in Fig. 2(d) the design was only slightly modified in order to fit into the original sample holder of the soft $\mathrm{x}$-ray ARPES end station.

\section{ACKNOWLEDGMENTS}

The authors are very grateful to Hans-Olof Larding of the former Kista workshop, Fritz Dubi of the PSI workshop, and Dr. Ray Barrett at the ESRF. This research has been supported by The Swedish Research Council (VR), the Göran Gustafsson Foundation, the Swiss National Science Foundation (through NCCR, MaNEP, and Grant No. 200020-105151), and the European Union.

\footnotetext{
${ }^{1}$ Y. Saitoh et al., Rev. Sci. Instrum. 71, 3254 (2000).

${ }^{2}$ C. Dallera, E. Puppin, G. Trezzi, N. Incorvaia, A. Fasana, L. Braicovich, N. B. Brookes, and J. B. Goedkoop, J. Synchrotron Radiat. 3, 231 (1996).

${ }^{3}$ U. Flechsig, L. Patthey, and T. Schmidt, AIP Conf. Proc. 705, 316 (2004); http://sls.web.psi.ch/view.php/beamlines/sis/index.htm

${ }_{5}^{4}$ J. Chang et al., Phys. Rev. B 75, 224508 (2007).

${ }^{5} \mathrm{M}$. Månsson et al. (unpublished).

${ }^{6} \mathrm{~J}$. Chang et al. (unpublished).
} 